\title{
QUALITÄTSBEWERTUNG FÜR ÖFFENTLICHE BIBLIOTHEKEN (ÖB) IN OBERÖSTERREICH
}

\author{
von Ulrike Reschitzegger
}

\section{Inhalt}

1. Geschichtlicher Abriss zum Öffentlichen Büchereiwesen in Österreich

2. Aktuelle Situation des Öffentlichen Büchereiwesens in Österreich

3. Ein kurzer statistischer Überblick

4. Erwachsenenbildung und Öffentliches Büchereiwesen

5. Das Qualitätssiegel des EB-Forum OÖ / Geschichte der Entwicklung

6. Aktuelle Situation

7. Der Weg zum Qualitätssiegel für öffentliche Bibliotheken in OÖ

7.1. Qualitätsbestätigung des EB-Forum OÖ für ÖB

7.2. Qualitätssiegel des EB-Forum OÖ für ÖB

8. Kriterienkataloge

8.1. Kriterienkatalog Qualitätsbestätigung

8.2. Kriterienkatalog Qualitätssiegel

9. Auditdurchführung

10. Voraussetzungen und Verantwortungen der AuditorInnen

Zusammenfassung: Bereits 1995 entwickelte die Landesarbeitsgemeinschaft „Erwachsenenbildungsforum Oberösterreich" (EB-Forum OÖ) differenzierte Qualitätsstandards für die gemeinnützigen Erwachsenenbildungseinrichtungen und für die öffentlichen Bibliotheken in Oberösterreich, deren Erfüllung die Qualität der Angebote der zertifizierten Institutionen garantiert und nach außen sichtbar macht. Seither wurden die Kriterienkataloge für das „Qualitätssiegel“ mehrmals evaluiert. In der letzten Überarbeitung wurde dem Qualitätssiegel für öffentliche Bibliotheken eine „Qualitätsbestätigung"vorangestellt, um allen öffentlichen Bibliotheken eine Bestätigung über ihre Qualität zu ermöglichen und diesen den Weg zum Qualitätssiegel transparenter zu gestalten. Die Inhalte der beiden Kriterienkataloge sowie der Ablauf von der Antragstellung bis zur Ausstellung einer Bestätigung oder eines Zertifikats werden dargestellt. Die beiden Kriterienkataloge wurden 2015 noch einmal überarbeitet, die Freigabe der Handbücher durch das EB-Forum OÖ ist mit Jahresbeginn 2016 terminiert.

Schlüsselwörter: Österreich; öffentliche Bibliotheken; EB-Forum OÖ; Qualitätssiegel; Qualitätsbestätigung; Qualitätssicherung; Qualitätsstandards; Audit; Evaluation 


\section{QUALITY ASSESSMENT FOR PUBLIC LIBRARIES IN UPPER AUSTRIA}

Abstract: As early as 1995 the working group on adult education „Erwachsenenbildungsforum Oberösterreich" (EB-Forum OÖ) in Upper Austria developed complex standards of quality on behalf of the nonprofit providers of adult education and of the public libraries in Upper Austria, which should guarantee and make visible the quality of the offerings of the certified institutions. Since then the sets of criteria have been evaluated for several occasions. With the last reworking there was put an affirmation of quality („Qualitätsbestätigung“) in front of the already existing quality label ("Qualitätssiegel") in order to allow all libraries to gain a confirmation of quality and to make the path leading to the quality label more transparent. This article presents both sets of criteria as well as the proceeding from putting forward a motion up to receiving one of both confirmations of quality. Both sets of criteria have been reworked 2015 and will be released by EB-Forum OÖ within the first months of 2016.

Keywords: Austria; Public Libraries; EB-Forum OÖ; Quality Label; Affirmation of Quality; Quality Management; Audit; Evaluation

\section{Geschichtlicher Abriss zum Öffentlichen Büchereiwesen in Österreich}

Der Beginn des öffentlichen Büchereiwesens in Österreich reicht zurück in das späte 19. und das beginnende 20. Jahrhundert mit der Errichtung von Volksbüchereien und Lesehallen, denen im Zusammenhang mit der aufsteigenden Volksbildungsbewegung eine zentrale Bedeutung zukommt. ${ }^{1}$ Im Austrofaschismus und dem nachfolgenden Nationalsozialismus wurden die Volks- und Arbeiterbüchereien aufgelöst bzw. kommunalisiert, deren Bestände "gesäubert" und schwarze Listen von verbotenen Autorlnnen angelegt. Nach 1945 war das österreichische Büchereiwesen fast völlig zerstört, nur mehr die Hälfte der rund 800 Büchereien vor 1933 konnten ihre stark dezimierten - und nationalsozialistisch verseuchten - Bestände wieder anbieten. Bis heute geblieben ist die Trennung nach kommunalen, kirchlichen und anderen Trägern wie Österreichischer Gewerkschaftsbund (ÖGB), Kammer für Arbeiter und Angestellte (AK) und Vereine. Geblieben ist auch der hohe Anteil ehrenamtlicher Mitarbeiterlnnen. Auch nach 1945 wurde das öffentliche Büchereiwesen dem Bereich der Erwachsenenbildung zugeordnet. 1973 bekannte sich die Bundesrepublik Österreich erstmals zur finanziellen Förderung der Erwachsenenbildung und formulierte ein „Bundesgesetz über die Förderung der Erwachsenenbildung und 
des Volksbüchereiwesens aus Bundesmitteln vom 21. März 1973“.²Allerdings räumt das Bundesgesetz keinen Anspruch auf die Gewährung einer Förderung ein, es ermöglicht der Republik Österreich lediglich, diese nach Maßgabe der Mittel finanziell zu unterstützen.

Eine ausführliche und kritische Darstellung des öffentlichen Bücherwesens findet sich auf der Website des Arbeitskreises kritischer Bibliothekarinnen und Bibliothekare - KRIBIBI. ${ }^{3}$

\section{Aktuelle Situation des öffentlichen Büchereiwesens in Österreich}

Das öffentliche Büchereiwesen in Österreich ist geprägt von zwei Organisationsformen: hauptberuflich und ehrenamtlich geführte öffentliche Bibliotheken (ÖB) in unterschiedlichen Trägerschaften.

Durch das Fehlen einer gesetzlichen Regelung über das Einrichten und Führen von ÖB gibt es für Gemeinden keine diesbezügliche Verpflichtung. Die Errichtung und Erhaltung ist noch immer eine freiwillige Leistung der Träger. Es verwundert unter diesen Umständen auch nicht, dass es in mehr als 50\% der Unterstützung österreichischen Gemeinden keine ÖB gibt. ${ }^{4}$ Für den Aufbau und die Modernisierung einer ÖB werden vom Bund und einzelnen Bundesländern unterschiedlich hohe finanzielle Mittel bereitgestellt. Das im Jahr 2010 für die ÖB zuständige Ministerium hat gemeinsam mit dem Büchereiverband Österreichs (BVÖ), dem Dachverband der ÖB, sieben österreichweit einheitliche Zielstandards und Förderungsrichtlinien festgelegt. ${ }^{5}$ Das Erreichen der in den Förderungsrichtlinien definierten Standards ist die Voraussetzung für die Förderungen der nun im Bundeskanzleramt angesiedelten Abteilung für das öffentliche Büchereiwesen. Auch die Bundesländer haben jeweils unterschiedliche Förderungsrichtlinien. ${ }^{6}$ Was fehlt, ist ein österreichweit gültiger Qualitätsstandard.

\section{Ein kurzer statistischer Überblick}

Die Statistik des BVÖ für das Jahr 2013 weist 1.449 öffentliche Bibliotheken in Österreich aus. Davon werden 1.173 ehrenamtlich geführt, das sind ca. 80\%. Diese ehrenamtlich geführten Bibliotheken sind durchwegs in ländlichen Gebieten sowie kleineren Gemeinden angesiedelt und bilden ein Netz für jenen Teil der Bevölkerung, der weit weg von größeren Städten wohnt. Die Stärken des Ehrenamtes sind Engagement und Begeisterung 
für diesen „Beruf“, die Schwächen sind wenige Öffnungszeiten, mangelndes Budget und oft viel zu kleine Räume. Aus der statistischen Erhebung des BVÖ geht hervor, dass zwar der Medienbestand aller ehrenamtlich geführten ÖB gleich groß ist wie in den hauptberuflich geführten, ein eklatanter Unterschied zeigt sich aber bei den Entlehnungen - ca. $25 \%$ bei den ehrenamtlich und ca. $75 \%$ bei den hauptamtlich geführten. Hier zeigt sich ganz deutlich, wie wichtig es wäre, durch hauptamtlich tätige Bibliothekarlnnen mehr Öffnungstage und -stunden anbieten zu können.

\section{Erwachsenenbildung und öffentliches Büchereiwesen}

Im Förderungsgesetz des Bundes werden die Mitgliedseinrichtungen der im Jahr 1972 gegründeten Arbeitsplattform „Konferenz der Erwachsenenbildung Österreichs" (KEBÖ) als vom Bund anerkannte und förderungswürdige Erwachsenenbildungsverbände genannt. Das öffentliche Büchereiwesen ist innerhalb der KEBÖ durch den BVÖ vertreten. ${ }^{7}$ Auf Initiative der Landesarbeitsgemeinschaften für Erwachsenenbildung und öffentliches Büchereiwesen - entsprechend der KEBÖ-Struktur - sowie der Länder wurde 2006 das Ländernetzwerk Weiter.Bildung ${ }^{8}$ mit den jeweils zuständigen Abteilungen bei den Ämtern der Landesregierungen und unter Einbindung der KEBÖ gegründet. Im Arbeitsprogramm für die Jahre 2006-2008 wird die Bedeutung der öffentlichen Bibliotheken auch durch den Absatz „Die Sicherstellung einer kontinuierlichen fachlichen Betreuung und Beratung, einer qualitätsvollen Aus- und Fortbildung für die Mitarbeiterlnnen oder elektronische Vernetzung der Medien- bzw. Buchbestände durch benutzerfreundliche elektronische Systeme muss gewährleistet werden" unterstrichen. ${ }^{9}$ Oberösterreich ist im Ländernetzwerk durch die Direktion Bildung und Gesellschaft sowie durch die Landesarbeitsgemeinschaft Erwachsenenbildungsforum Oberösterreich (EB-Forum OÖ) vertreten. ${ }^{10}$

\section{Das Qualitätssiegel des EB-Forum OÖ für öffentliche Büchereien / Geschichte der Entwicklung}

Als Ergebnis des 1995 vom EB-Forum OÖ in Auftrag gegebenen und vom „market - Institut“ durchgeführten Projekts „Planquadrat 2001. Weiterbildung in OÖ“ entwickelten die Mitgliedseinrichtungen differenzierte Qualitätsstandards für die gemeinnützigen Erwachsenenbildungseinrichtungen und für die öffentlichen Bibliotheken. Bereits 1998 wurde den geprüften 
und auditierten Mitgliedseinrichtungen des EB-Forum OÖ erstmals das „Qualitätssiegel der oö. Erwachsenen- und Weiterbildungseinrichtungen“ in einem feierlichen Rahmen von Bundesministerin Elisabeth Gehrer und Landeshauptmann Dr. Josef Pühringer verliehen. Gefördert wird das Vorhaben seit Anbeginn vom Land OÖ und bis 2012 vom jeweiligen Unterrichtsressort ${ }^{11}$, zuletzt vom Bundesministerium für Unterricht, Kunst und Kultur. Ein ganz wichtiger Aspekt dabei war die Transparenz der Informationen über die jeweilige Bildungseinrichtung und deren Angebote nach innen und nach außen für Interessierte und Teilnehmerlnnen sowie für Benutzerlnnen und Besucherlnnen einer öffentlichen Bibliothek.

\section{Aktuelle Situation}

Im Zuge der letzten Evaluierung des Qualitätssiegels für ÖB wurde der Kriterienkatalog grundlegend überarbeitet und den Anforderungen an eine moderne und qualitätsgesicherte Bibliotheksarbeit angepasst. Weiters wurde der Auditierungsablauf modifiziert. Dafür wurde das in Österreich gültige Zertifizierungsverfahren für Qualitätsmanagementsysteme auf Grundlage der Ö-Norm EN ISO 9001 herangezogen und mit den wesentlichen Kriterien der Bildungseinrichtungen und der öffentlichen Bibliotheken ergänzt. Expertinnen und Experten aus dem Bibliothekswesen waren in den Prozess der Evaluierung und Überarbeitung einbezogen.

Bedingt durch die schwierigen Rahmenbedingen und unterschiedlichen Arbeitsbedingungen in den ÖB gibt es enorme Qualitätsunterschiede. Um aber allen ÖB in OÖ eine Überprüfung ihrer Qualität und in Folge den Zugang zum Qualitätssiegel zu ermöglichen, wurde ein zusätzlicher Nachweis im Rahmen einer Qualitätsbestätigung entwickelt. Damit wurde der Weg zum Qualitätssiegel für ÖB transparenter und nachvollziehbarer gestaltet.

\section{Der Weg zum Qualitätssiegel für öffentliche Bibliotheken in OÖ}

Wie bereits erwähnt gibt es in Österreich keine verbindlichen und umfassenden Qualitätsstandards und -kriterien für ÖB außer jenen, die zur Erfüllung von Förderungen des Bundes und der Bundesländer jeweils notwendig sind. Die den Förderungsrichtlinien des Bundes zugrundeliegenden Standards sind denen der beiden oberösterreichischen Qualitätsnachweise ähnlich, aber nicht kongruent. 


\subsection{Qualitätsbestätigung}

Das für die Qualitätsbestätigung des EB-Forum OÖ erstellte Onlinetool ermöglicht den Bibliothekarlnnen, in einem ersten Schritt selbst zu überprüfen, ob und welche der vorgegebenen Kriterien bereits teilweise oder voll erfüllt werden. Begleitet, beraten und unterstützt werden sie dabei durch Auditorlnnen und/oder FachexpertInnen aus dem Bibliotheksbereich. Die Kriterien für die Qualitätsbestätigung erarbeitete die Arbeitsgruppe „Evaluierung für öffentliche Bibliotheken im EB-Forum OÖ“.

\section{Aufbau Qualitätsbestätigung}

Die Qualitätsbestätigung wurde als Vorstufe für das Qualitätssiegel entwickelt, Kernstück ist das Selbstbewertungstool. Dafür wurde eine Checkliste erarbeitet und die Fragen so formuliert, dass sie auch für Personen ohne Vorkenntnisse im Umgang mit Qualitätsmanagement verständlich sind.

Der Ablauf wurde in mehrere Module geteilt:

- Selbstbewertung der ÖB mittels digitaler Checkliste, die ausgefüllt wieder an die zuständige Auditorln / Fachexpertln retourniert wird

- Ermittlung der vorgegebenen Kennzahlen anhand der Jahresmeldungen (Land OÖ oder BVÖ) der letzten 3 Jahre durch Auditorln / Fachexpertln

- Je nach Ergebnis ist ein Kurzaudit vorgesehen

- Bericht und Übersicht der Punktevergabe als Grundlage für die Ausstellung der Qualitätsbestätigung

- Nach Erreichung einer vorgegeben Punktezahl bzw. Prozentzahl und eines positiven Kurzaudits wird über die Qualitätsprüfung eine Qualitätsbestätigung ausgestellt, die drei Jahre gültig ist.

\subsection{Qualitätssiegel}

Nach Ausstellung der Qualitätsbestätigung und Erfüllung der Grundanforderungen für die Vorprüfung kann um das Qualitätssiegel angesucht werden. Nach einem positiven Ergebnis aus dem Audit wird die Ausstellung des EB-Qualitätssiegel bei der Auditkommission beantragt. Nach positiver Prüfung wird dieses ausgestellt und ist für drei Jahre gültig. Die Bibliothek verpflichtet sich dabei, in einer nachweislichen Form das Qualitätsmanagement weiter zu führen, die Einhaltung der Kriterien intern zu überprüfen und die eigenen sowie die Kundlnnenbeziehungsprozesse zu evaluieren und Verbesserungen durchzuführen. 
Öffentliche Bibliotheken können die Qualitätsbestätigung oder das Qualitätssiegel beantragen, wenn eine Förderfähigkeit durch das Land OÖ gegeben ist. Für Bibliotheken, die bereits über das Qualitätssiegel verfügen, wurde eine Übergangsregelung festgelegt. Zuständig für Organisation und Abwicklung ist das Projektbüro des EB-Forum OÖ.

Alle Informationen zu den beiden Modellen Qualitätsbestätigung und Qualitätssiegel sind in den jeweiligen Handbüchern „Qualitätsstandard für öffentliche Bibliotheken in Oberösterreich "12 veröffentlicht. Die beiden Handbücher sind einerseits eine Orientierungsgrundlage für die Mitarbeiterlnnen in den ÖB und andererseits ein Arbeitsinstrument für die Auditorlnnen.

Beiden Modellen gemeinsam sind die Qualitätsbereiche:

- Institutionelle und organisatorische Kriterien

- Personelle Rahmenbedingungen / Bibliothekarische Aus-, Fort- und Weiterbildung

- Bibliotheksangebote und Veranstaltungen

- Auftritt der Bibliothek bzw. Informationen nach außen

- Bibliotheksräume und Ausstattung

\section{Kriterienkataloge}

\subsection{Kriterienkatalog Qualitätsbestätigung}

Der Kriterienkatalog zur Qualitätsbestätigung für ÖB umfasst insgesamt 15 Qualitätsbereiche und ist Teil des Handbuches „Qualitätsstandard für öffentliche Bibliotheken in Oberösterreich, Qualitätsbestätigung“, Juni 2014. Die folgende Übersicht ist eine verkürzte - aber dennoch korrekte - Darstellung:

I. Checkliste zur Selbstbewertung der öffentlichen Bibliothek

1 Organisation der Bibliothek Aktuelle Informationen über die ÖB / Dokumentation relevanter Bibliotheksdaten

2 Informationsmöglichkeit für Interessierte und MitarbeiterInnen Bibliotheksordner / Schaukasten / Eingangsbereich /regelmäßige Informationen und Berichte in Printmedien / Webseite / eigene Homepage / Zielplanung / Stellenbzw. Aufgabenbeschreibungen / Fort- und Weiterbildungspläne

3 Bibliothekarische Aus-, Fort- und Weiterbildung der MitarbeiterInnen Bibliothekarische Qualifikationen / Dokumentation absolvierter Fort- und Weiterbildungen

4 Ziele/ Zielerreichung/ Zielüberprüfung Zielfestlegungen messbar und terminiert / Dokumentation Zielerreichung / Korrekturmaßnahmen 
5 EDV Hard- und Software

Verwaltung der Medien, Software / Internetarbeitsplatz, -plätze /OPAC / OnlineKatalog / Web-OPAC mit Zusatzfunktionen (z. B. Vorbestellung, Reservierung)

6 Systematik

Verwendete Systematik zur Erschließung der Medien / ÖSÖB bis zu welcher Stufe / Mischformen etc.

7 Veranstaltungen Planung / Durchführung / Methoden der Dokumentation

II. Checkliste für das Kurzaudit mit Fachgespräch zur Bewertung durch die Auditorlnnen

8 Auffindbarkeit der Öffentlichen Bibliothek Hinweistafeln im Ort / Beschilderung Haus / Barrierefreiheit

9 Informationen nach außen (Schaukasten, Türe, Fenster)

10 Eindruck beim Eintreten Helligkeit der Räume / einladende Gestaltung / Raumtemperatur

11 Gestaltung der Bibliothek Einrichtung / Beleuchtung / Regale / Orientierung für BenutzerInnen

12 Datenmaterial liegt vor Einwohnerlnnenzahl / Entlehnungen /Raumgröße $\left(\mathrm{qm}^{2}\right)$ / Öffnungstage und -stunden / Zahlen zu BenutzerInnen und BesucherInnen

13 Bestand (Medienbestand der letzten drei Jahre) Bewertung Bestand / Mediengruppen / Bestandsergänzung

14 Weitere Kennzahlen der Bibliothek

Medienumschlagszahl (MUZ) / Erneuerungsquote / Benutzeranteil in \% / Besucheranteil absolut / Öffnungszeiten und Öffnungstage / Quadratmeter pro 1000 Medien 15 Fachgespräch innerhalb des Kurzaudits

Inhalte gewählt aus dem aktuellen Ergebnis der Selbstevaluation der Bibliothek, Vergabe von Bonuspunkten aus dem Fachgespräch ist möglich

Den 15 Qualitätsbereichen des Kriterienkatalogs zur Qualitätsbestätigung sind 45 Kriterien zugeordnet, 18 davon sind unbedingt positiv nachzuweisen. Insgesamt können maximal 93 Punkte vergeben werden. Für ein positives Ergebnis zur Ausstellung einer Qualitätsbestätigung müssen neben dem erfolgreich bewerteten Fachgespräch im Kurzaudit mindestens $75 \%$ der maximalen Punktezahl bzw. 70 Punkte erreicht werden.

\subsection{Kriterienkatalog Qualitätssiegel}

Der Kriterienkatalog zum Qualitätssiegel für ÖB umfasst fünf Qualitätsbereiche und ist Teil des Handbuches „Qualitätsstandard für öffentliche 
Bibliotheken in Oberösterreich, Qualitätssiegel“, Neufassung Juni 2014. Den Qualitätsbereichen sind jeweils unterschiedlich viele Kriterien mit Festlegung der Punkte und Erläuterung der Punktevergabe zugeordnet. Die folgende Übersicht ist eine verkürzte - aber dennoch korrekte - Darstellung des Kriterienkataloges:

1 Institutionelle und organisatorische Kriterien

1.1 Die Öffentliche Bibliothek ist im EB-FORUM OÖ vertreten

1.2 Organigramm > Datum und Unterschrift, Kenntnisnahme durch Träger, Darstellung Umfeld der ÖB

1.3 Leitbild > Kenntnisnahme Träger, interne Kommunikation

1.4 Ziele > aus Leitbild ableitbar, durch Träger freigegeben, interne Kommunikation, Zielreflexion

2a Personelle Rahmenbedingungen a) hauptamtlich geführte ÖB

2.1a Stellen-/Arbeitsplatzbeschreibungen > Kenntnisnahme durch Träger

2.2a Bibliothekarische Qualifikation und Berufserfahrung > Leitung und MitarbeiterInnen

2.3a Kriterien der Aufnahme neuer MitarbeiterInnen

2.4a MitarbeiterInnengespräch > Vereinbarungen zu Fort- und Weiterbildungen

2.5a Fort- und Weiterbildungen > Dokumentation

2b Personelle Rahmenbedingungen b) ehrenamtlich geführte ÖB

2.1b Stellenbeschreibung Leitung > Kenntnisnahme durch Träger

2.2bBibliothekarische Qualifikation und Berufserfahrung > Leitung und Mitarbeiterlnnen

2.3b Kriterien für die Aufnahme neuer MitarbeiterInnen

2.4bStrukturierte Gespräche > Feedback-, Ein- und Ausstiegsgespräche

2.5b Fort- und Weiterbildungen > Planung, Umsetzung, Dokumentation

3 Bibliotheksangebote

3.1 Literatur- und Medienangebot und -vielfalt

3.1.1 Bestandsgröße und Bestandskonzept

3.1.2 Bestandverteilung

3.2 Aktualisierung des Medienbestandes > Methoden

3.3 Verwaltung des Bestandes - technische Mittel > Zugriff auf den Bestand für Benutzerlnnen

3.4 Statistische Daten > Dokumentation

3.5 Medienumschlagszahl > entsprechend dem Qualitätsstandard des EB-Forum OÖ

3.6 Bestandsergänzung - Aktualität des Medienbestandes (schriftliche Nachvollziehbarkeit)

3.7.1 Zahl der BenutzerInnen 
3.7.2 Zahl der BesucherInnen > Methoden der Erhebung

3.8 Veranstaltungen > Planung, Durchführung, Dokumentation

4 Auftritt der Bibliothek

4.1 Transparenz der Informationen zur öffentlichen Bibliothek > Organigramm, Leitbild, Kontaktdaten

4.2 Öffnungszeiten > entsprechend dem Qualitätsstandard des EBForum OÖ

4.3 Veröffentlichung der Öffnungszeiten > in welcher Form,

4.4 Benutzungs- und Gebührenordnung > Inhalte, Zugänglichkeit für Benutzerlnnen

4.5 Systematik des Medienbestandes > Wahl der Systematik, Aufstellung und Präsentation der Medien

5 Bibliotheksräume und Ausstattung

5.1.1 Auffindbarkeit der öffentlichen Bibliothek > Wegweiser, Beschilderung

5.1.2 Zugang zur öffentlichen Bibliothek > Barrierefreiheit

5.2 Raumgröße > entsprechend dem Qualitätsstandard des EB-Forum OÖ

5.3 Ausstattung der Bibliotheksräume

Für die 5 Qualitätsbereiche des Qualitätssiegels mit insgesamt 27 Kriterien können maximal 91 Punkte erreicht werden. 22 Kriterien davon sind unbedingt positiv nachzuweisen. Für ein positives Gesamtergebnis müssen mindestens $85 \%$ bzw. 77 Punkte erreicht werden.

\section{Auditdurchführung}

Rahmenbedingungen

Für ein Audit ist das Vier-Augen-Prinzip vorgesehen. Die jeweils für die Durchführung des Audits eingesetzten Auditorlnnen werden dazu schriftlich beauftragt. In der Beauftragung sind die Rahmenbedingungen festgelegt und mit der Unterschrift der AuditorInnen wird Unparteilichkeit, Datenschutz und Vertraulichkeit zugesichert.

Arbeitsunterlagen

Folgende Arbeitsunterlagen stehen den Auditorlnnen zur Verfügung: die Unterlagen aus der Vorprüfung durch das Projektbüro, das jeweils aktuelle Handbuch, eine Checkliste zur Mitschrift beim Audit und Vorlagen für das Auditprotokoll sowie für den Auditbericht. 


\section{Punktevergabe}

Die Punktevergabe ist aufbauend festgelegt und im Kriterienkatalog dargestellt. Die Auswahl, welche Unterlagen pro Kriterium als Nachweis der Erfüllung eingesehen und für den Bericht dokumentiert werden, liegt in der Verantwortung der AuditorInnen. Die Dokumentation muss Art der Unterlage, den genauen Titel, wichtige Inhalte dazu sowie Datum und Umfang beinhalten. Es ist wichtig und notwendig, über das Auditprotokoll und/ oder den Auditbericht die Punktevergabe nachweisbar zu dokumentieren.

\section{Voraussetzungen und Verantwortungen für Auditorlnnen}

Im Handbuch sind die Voraussetzungen für die Tätigkeit als Auditorln festgelegt:

- Fachkenntnisse im Bibliothekswesen

- Kenntnisse Qualitätsmanagement

- Einschulung oder Zertifizierung als Auditorln

\section{Beispiel für die Punktevergabe aus dem Kriterienkatalog Qualitätssiegel zur Verdeutlichung}

1.4 Sind die Ziele der Öffentlichen Bibliothek aus dem Leitbild ableitbar, durch Budgetplanung realistisch sowie durch den/die Träger bestätigt bzw. freigegeben? In welcher Form werden die Ziele mit den Mitarbeitern/innen kommuniziert?

Reflexion der Zielerreichung / Die Kommunikation mit den Mitarbeiterlnnen ist abgestuft auf die Tragweite ihrer Aufgabenbereiche und ihrer Zuständigkeiten zu verstehen

- aktuelle Jahresziele und diskursive Darstellung der Zielerreichung 1 Punkt

- Terminierte und messbare aktuelle Jahresziele und mittelfristige Planung (mindestens 3 messbare Ziele), schriftliche Dokumentation der Zielerreichung - 2 Punkte

- Jahresziele, mittelfristige Planung und Veranstaltungsplanung (mindestens 5 messbare Ziele) Schriftliche Dokumentation: Soll-Ist-Vergleich mit Zielreflexion - 3 Punkte

Ziele bzw. Pläne können sein:

Bestandsbereinigung oder -veränderung (wegen Raumgröße, veraltetem Bestand, veralteten Medien z.B. CDs etc.), Einführung von e-books / Ko- 
operation mit Kindergarten (Bücher dem Kindergarten zur Verfügung zu stellen oder Animationen mit Bilderbüchern, Besuche vereinbaren etc.) und mit Vereinen - Bücherstand bei Vorträgen, gemeinsame Literaturabende organisieren etc.) / Planung von Veranstaltungen - welche, wann, mit wem / Modernisierung der Ausstattung (Möbel, Hard- oder Software).

Für eine Punktevergabe müssen die einzelnen Fragen jeweils positiv beantwortet und die geforderten Unterlagen zur Bestätigung vorgelegt werden. Die Einschätzung, ob die Antworten ausreichend beantwortet bzw. nachvollziehbar erläutert wurden und die Nachweise bzw. Dokumentationen den Anforderungen entsprechen, obliegt den Auditorlnnen.

Aktueller Stand Juli 2015: Die Kriterienkataloge und die Handbücher Qualitätsbestätigung und Qualitätssiegel für ÖB wurden ab Herbst 2014 in einer neuen Arbeitsgruppe überarbeitet, die Freigabe durch das EB-Forum OÖ ist für die Generalversammlung 2016 geplant.

Die Projektleitung für den gesamten Bereich Qualitätssiegel des EB-Forum OÖ wurde dem Institut für Berufs- und Erwachsenenbildungsforschung (IBE), Weingarts-

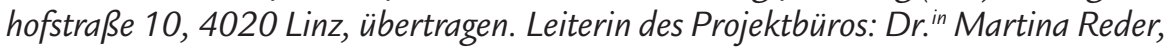
E-Mail: reder@ibe.co.at

Ulrike Retschitzegger Ausbildung BVÖ zur hauptamtlichen Bibliothekarin für öffentliche Bibliotheken Zertifizierte Auditorin gemäß ISO 19011 Auditorin für das Qualitätssiegel des EB-Forum OÖ E-Mail: ulrike.retschitzegger@gmx.at

\section{Literatur}

Handbuch „Qualitätsstandard für öffentliche Bibliotheken in Oberösterreich, Qualitätsbestätigung“, Juni 2014, Erwachsenen- und Weiterbildungsforum Oberösterreich, p.A. Mag. Harald Wolfslehner, 4021 Linz, Wiener Straße 150.

Handbuch „Qualitätsstandard für öffentliche Bibliotheken in Oberösterreich, Qualitätssiegel“, Neufassung Juni 2014, Verfahren und Kriterien, Erwachsenen- und Weiterbildungsforum Oberösterreich, p.A. Mag. Harald Wolfslehner, 4021 Linz, Wiener Straße 150.

Stifter, Christian: „Library work is not philanthropy“. Zur historischen Rolle der Volksbüchereien im Kontext der Volksbildung im 19. und 20. 
Jahrhunderts. In: Zur Geschichte der öffentlichen Bibliotheken in Österreich. Hrsg. Alfred Pfoser und Peter Vodosek, BVÖ-Materialien 2. Wien, Büchereiverband Österreichs, 1995, S. 70-78.

LÄNDER-NETZWERK WEITER.BILDUNG. Eine Initiative der Landesarbeitsgemeinschaften für Erwachsenenbildung und öffentliches Büchereiwesen sowie der Länder. Grundsatzerklärung und Arbeitsprogramm für die Jahre 2006 bis 2008. http://www.pfiffikus.at/Inw/doc/Papier\%20L\%C3\%A4nder-Netzwerk\%2020.3.2006.pdf (27.09.2012)

Arbeitskreis kritischer Bibliothekarinnen und Bibliothekare, Bibliothekswesen in Österreich http://www.kribibi.at/bibliothekswesen (27.09.2012)

1 Stifter, Christian: „Library work is not philantropy“. In: Zur Geschichte der öffentlichen Bibliotheken in Österreich, Wien 1995, S. 70-87. http:// www.adulteducation.at/de/literatur/textarchiv/653/

2 http://erwachsenenbildung.at/themen/eb in oesterreich/gesetze/ foerderungsgesetz.php

3 Öffentliche Büchereien in Österreich, http://www.kribibi.at/bibliothekswesen

4 Ebenda, Absatz 8.

5 www.bvoe.at/oeffentliche bibliotheken/daten und fakten/zielstandards und www.kunstkultur.bka.gv.at/site/8005/default.aspx

6 www.bvoe.at/serviceangebote/foerderungen

7 http://erwachsenenbildung.at/themen/eb in oesterreich/organisation/keboe.php

8 http://erwachsenenbildung.at/themen/eb in oesterreich/organisation/laender.php

9 LÄNDER- NETZWERK WEITER.BILDUNG, Arbeitsprogramm für die Jahre 2006 bis 2008, Seite 4 von 11. http://www.pfiffikus.at/Inw/doc/ Papier\%20L\%C3\%A4nder-Netzwerk\%2020.3.2006.pdf

10 http://www.weiterbilden.at/index.php/eb-forum

11 Handbuch Qualitätssiegel der Oberösterreichischen Erwachsenen- und Weiterbildungseinrichtungen, S. 5. http://www.ibe.co.at/fileadmin/ AblageBox/EBQS BQS/EBQS/EBQ Handbuch 2014.pdf

12 Handbücher erhältlich über das EBQ-Projektbüro, Projektleitung: Horst Harald Geyer (BBRZ / EB-Forum OÖ), Sekretariat: Elke Kagerer, Tel. +43 (0)732 / 6922-5255

(Alle Fußnoten wurden am 27.09.2014 überprüft.)

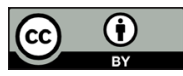

Dieses Werk ist lizenziert unter einer Creative-Commons-Lizenz Namensnennung 4.0 International 